\title{
Circadian Control of Neurogenesis
}

\author{
Erin M. Goergen, Leslie A. Bagay, Kris Rehm, Jeanne L. Benton, Barbara S. Beltz \\ Department of Biological Sciences, Wellesley College, Wellesley, Massacusetts 02481
}

Received 29 May 2002; accepted 29 May 2002

\begin{abstract}
The life-long addition of new neurons has been documented in many regions of the vertebrate and invertebrate brain, including the hippocampus of mammals (Altman and Das, 1965; Eriksson et al., 1998; Jacobs et al., 2000), song control nuclei of birds (Alvarez-Buylla et al., 1990), and olfactory pathway of rodents (Lois and Alvarez-Buylla, 1994), insects (Cayre et al., 1996) and crustaceans (Harzsch and Dawirs, 1996; Sandeman et al., 1998; Harzsch et al., 1999; Schmidt, 2001). The possibility of persistent neurogenesis in the neocortex of primates is also being widely discussed (Gould et al., 1999; Kornack and Rakic, 2001). In these systems, an effort is underway to understand the regulatory mechanisms that control the timing and rate of neurogenesis. Hormonal cycles (Rasika et al., 1994; Harrison et al., 2001), serotonin (Gould, 1999; Brezun and Daszuta, 2000; Beltz et al., 2001), physical activity (Van Praag et al., 1999) and living conditions (Kemperman and Gage, 1999; Sandeman and Sandeman, 2000) influ-
\end{abstract}

ence the rate of neuronal proliferation and survival in a variety of organisms, suggesting that mechanisms controlling life-long neurogenesis are conserved across a range of vertebrate and invertebrate species. The present article extends these findings by demonstrating circadian control of neurogenesis. Data show a diurnal rhythm of neurogenesis among the olfactory projection neurons in the crustacean brain, with peak proliferation during the hours surrounding dusk, the most active period for lobsters. These data raise the possibility that light-controlled rhythms are a primary regulator of neuronal proliferation, and that previously-demonstrated hormonal and activity-driven influences over neurogenesis may be secondary events in a complex circadian control pathway. () 2002 Wiley Periodicals, Inc. J Neurobiol 53: 90-95, 2002

Keywords: circadian; diurnal; neurogenesis; serotonin; Crustacea; olfactory

\section{INTRODUCTION}

Persistent neurogenesis among sensory and interneuronal populations in the olfactory pathway of a variety of decapod crustacean species has been intensively studied (Harzsch and Dawirs, 1996; Sandeman et al., 1998; Harzsch et al., 1999; Schmidt, 2001). After

Correspondence to: B. S. Beltz (Bbeltz@wellesley.edu).

Contract grant sponsor: National Science Foundation; contract grant number: IBN 0091092.

Contract grant sponsor: Howard Hughes Medical Institute Undergraduate Biological Sciences Education; contract grant number: 52003001.

Contract grant sponsor: National Science Foundation Site Grant; contract grant number: DBI-0097499.

(C) 2002 Wiley Periodicals, Inc.

Published online in Wiley InterScience (www.interscience.wiley.com) DOI 10.1002/neu.10095 larval life in the American lobster (Homarus americanus), continued neurogenesis in the central nervous system is found only among the local and projection neurons of the olfactory pathway, and neurogenesis persists in these regions throughout the lobster's life (Fig. 1) (Harzsch et al., 1999). The sensory, local, and projection neurons of the crustacean brain are functionally analogous to groups of neurons in the olfactory system of adult vertebrates that have a similar capacity for life-long neurogenesis (Lois and AlvarezBuylla, 1994; Graziadei and Monti Graziadei, 1986). Although the crustacean olfactory pathway has a functional anatomy that is parallel to the vertebrates (Strausfeld and Hildebrand, 1999), its organization provides experimental advantages because neuronal somata are arranged in anatomically distinct clusters according to their function. Thus in crustaceans, the 


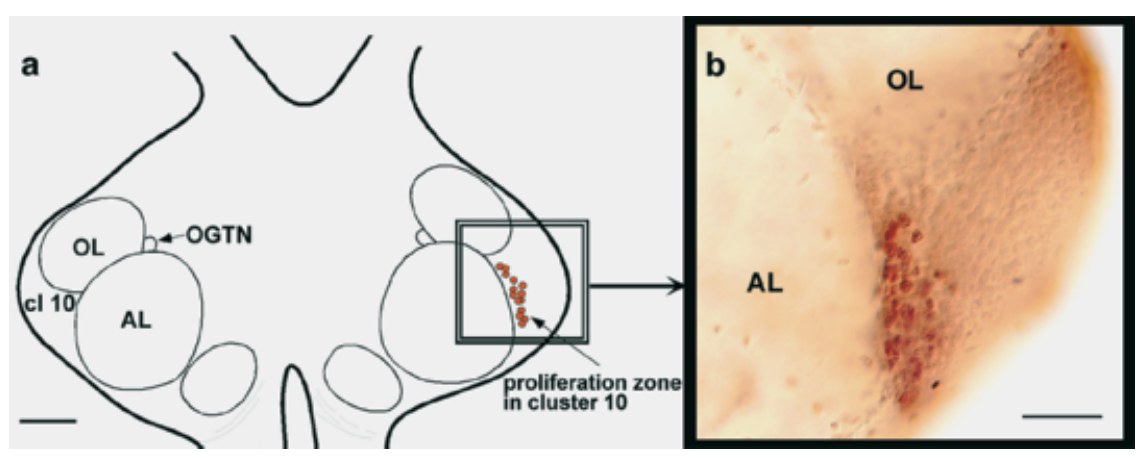

Figure 1 (a) Schematic diagram of the brain of Homarus americanus, illustrating the positions of deutocerebral synaptic areas. The olfactory pathway includes the paired olfactory lobes (OLs), accessory lobes (ALs), and olfactory globular tract neuropils (OGTNs). Cell cluster 10 (cl 10) contains the cell bodies of the deutocerebral projection neurons. The region outlined on the right side of the brain indicates the position of the neuronal proliferation zone in cell cluster 10. Scale $=200$ $\mu \mathrm{m}$. (b) Photo of BrdU-labeled neurons (darkly stained cells) in the proliferation zone of cluster 10.

Scale bar $=50 \mu \mathrm{m}$.

somata of local interneurons are found in clusters medial to the primary olfactory processing area, the olfactory lobe (OL), while projection neurons are located lateral to the OLs in a distinct cluster (cluster 10; Fig. 1). The deutocerebral projection neurons of cluster 10, which are the primary focus of this article, innervate the OL, accessory lobe (AL) (Sandeman et al., 1995) and olfactory globular tract neuropil (OGTN) (Sandeman and Sandeman, 1994), and possess axons which project to protocerebral synaptic areas in the eyestalk (Fig. 1).

\section{METHODS}

Because neurogenesis in the crustacean olfactory pathway is known to fluctuate during the molt cycle (Harrison et al., 2001), only animals in intermolt stages (Aiken, 1973) were used for the present circadian study of neuronal proliferation. Juvenile lobsters $(50-100 \mathrm{mg}$ ) for the "normal light/ dark (LD)" experiment were kept under a 12:12 h light:dark cycle (light levels at 6-10 $\mu$ Einsteins $/$ meter $^{2} \mathrm{~s}$ at the surface of the water) for 2 weeks prior to assessment of neuronal proliferation by in vivo incorporation of bromodeoxyuridine (BrdU). During that period, animals were fed every 3 days within the dusk sampling period (clock time 16:30-19:30). Twenty four hours prior to BrdU incubation, animals were placed individually into small diameter tubes that restricted movement. For each of the six sampling times (Fig. 2), five animals were incubated in $0.2 \mathrm{mg} \mathrm{BrdU} / \mathrm{ml}$ seawater for $3 \mathrm{~h}$. This protocol therefore sampled proliferative activity for $18 \mathrm{~h}$ out of a 24-h period. Prior work (Harzsch and Dawirs, 1996; Harzsch et al., 1999; Benton and Beltz, 2002) established that the BrdU concentration used results in intense labeling of a maximum number of cells. The 3-h incubation time was chosen based on the necessity to resolve the BrdU incorporation at various time intervals during a $24-\mathrm{h}$ period. Studies in crustaceans also have shown that the cell cycle of postembryonic neuroblasts is $2-3 \mathrm{~h}$ (Harzsch and Dawirs, 1996). Thus, the chosen incubation time was sufficient to capture a complete cell cycle.

Following BrdU incubation, lobsters were immediately decapitated and heads were immersed in $4 \%$ paraformaldehyde in $0.1 M$ phosphate buffer, $\mathrm{pH} 7.4$ to prevent further neurogenesis.

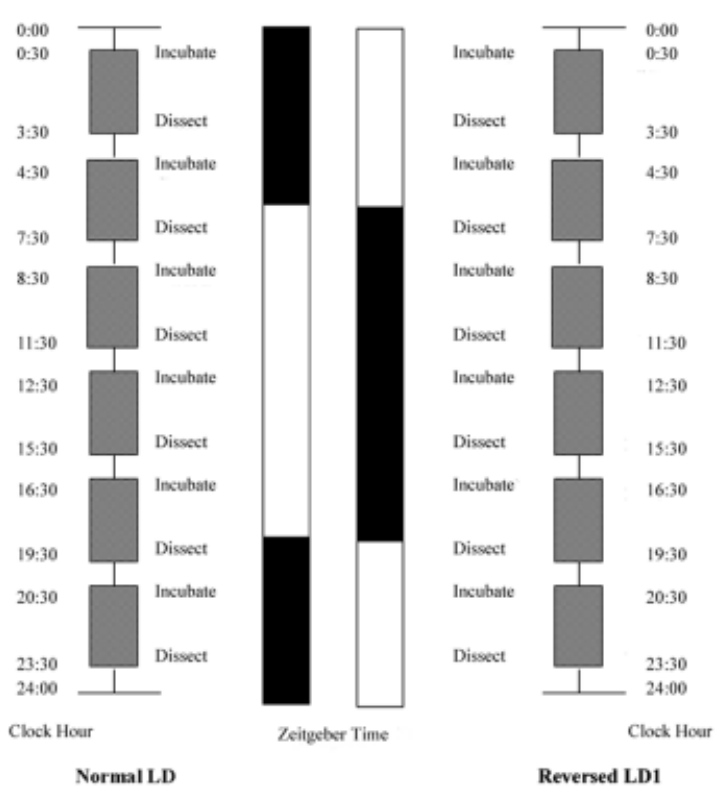

Figure 2 Summary of methods used for experiments testing the circadian influence on neurogenesis. BrdU incubation and dissection times are indicated (outer bars) relative to the clock hour and zeitgeber time (inner bars) for normal LD and reversed LD1 experiments. 

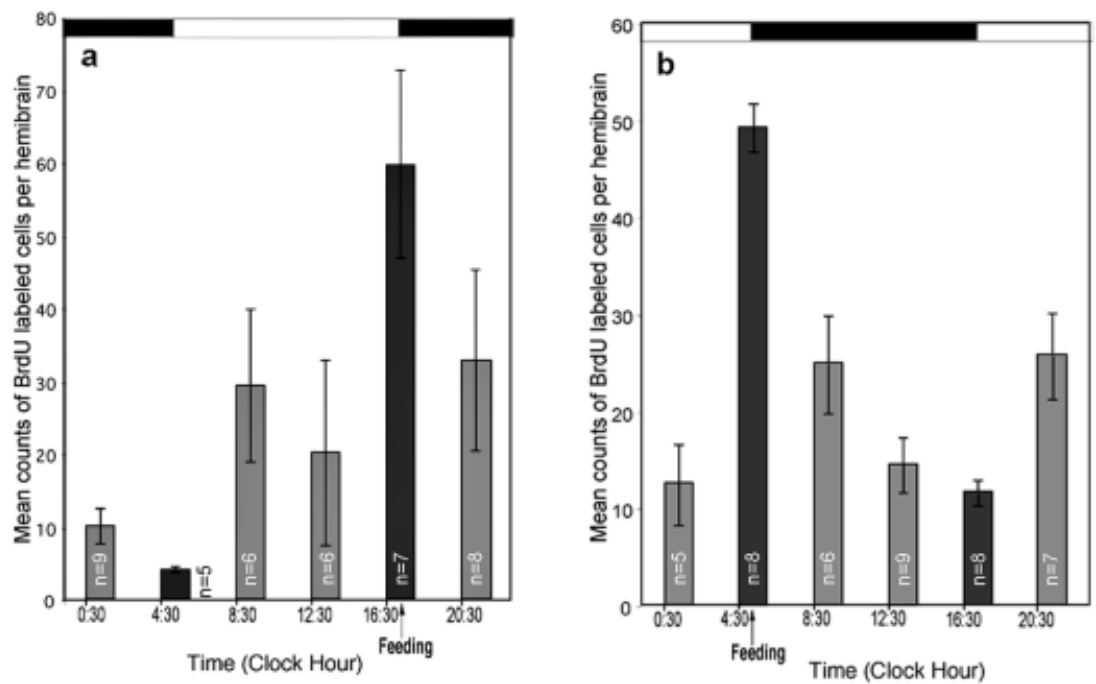

Figure 3 Graph of mean counts ( \pm SEM) of BrdU-labeled neurons in the projection neuron cluster relative to time of day (clock hour) for lobsters maintained on the normal LD light/dark cycle (a), and on the reversed LD1 light/dark cycle (b). The darker vertical bars indicate the 3-h sampling periods at dawn and dusk. The numbers of samples assessed for each time point are indicated within the respective vertical bars. The horizontal band on top of this graph indicates illumination conditions (white, light; black, dark).

The brains were then dissected free of the head capsule, and placed in $4 \%$ paraformaldehyde fixative for $12-20 \mathrm{~h}$. Brains were processed as whole mounts by standard immunocytochemical methods for detection of BrdU using horseradish peroxidase-coupled antibodies for visualization of the nucleotide label (Harzsch et al., 1999). Brains were examined using a Nikon compound microscope, and labeled neurons in cluster 10 were counted blindly using a camera lucida attachment. Although specific neuronal markers are not available for crustacean neurons, the identity of the BrdU labeled cells as projection neurons was previously established based upon cell size, shape, and position in the cell cluster (Harzsch et al., 1999).

Differences among sampling times for the normal LD and reversed LD1 experiments were assessed by a one-way analysis of variance (ANOVA). Differences between individual sampling times were then assessed using Student's $t$ test.

\section{RESULTS}

In the normal LD experiment, BrdU-labeled nuclear profiles were found within the proliferation domain of the projection neuron cluster (cluster 10) for all time points sampled. The numbers of labeled cells showed a diurnal rhythmicity, with $38.3 \%$ of all labeled neurons being born in the 3-h period around dusk [Fig. $3(a)]$. The fewest labeled profiles were seen during the 3-h period surrounding dawn.

If a biological rhythm is endogenous, it will be maintained under constant conditions. Therefore, to test whether the normal LD rhythm of neurogenesis is due to an endogenous oscillator, animals that had been in a normal LD cycle for 2 weeks were placed in total darkness for 3 days ("normal DD"). Lobsters were not fed during the DD period. When proliferation during the 3-h periods surrounding dawn and dusk was assessed after 3 days in constant darkness, the relationship between the numbers of BrdU-labeled profiles was the same as in the normal LD experiment (Table 1). The major peak of neurogenesis was found at dusk, and the number of labeled cells was not diminished by the 3-day period of darkness.

If a biological rhythm is entrained by an external zeitgeber, a change in the zeitgeber stimulus will result in changes in timing of the rhythm. Therefore, to test whether light is a zeitgeber for neurogenesis, another group of animals ("reversed LD1") was kept under a light cycle that was shifted by 12 h (Fig. 2), and animals were fed every 3 days within the 3 -h sampling period around subjective dusk. In animals exposed to the reversed light conditions for 2 weeks, the peak period of neurogenesis was phase shifted so that the largest number of BrdU-labeled profiles was generated during the 3-h period surrounding subjective dusk [Fig. 3(b)]. As in the normal LD study, the fewest labeled cells were generated in the $3 \mathrm{~h}$ surrounding dawn.

Reversed LD1 animals that were subsequently 
Table 1 Dawn and Dusk Results for All Experiments

\begin{tabular}{|c|c|c|c|c|}
\hline Treatment & $\begin{array}{c}\text { Light Condition } \\
\text { 6:00 18:00 }\end{array}$ & $\begin{array}{l}\text { Labeled Profiles } \\
\quad(4: 30-7: 30)\end{array}$ & $\begin{array}{c}\text { Labeled Profiles } \\
(16: 30-19: 30)\end{array}$ & $\begin{array}{c}p \text {-value } \\
\text { (Dawn vs. Dusk) }\end{array}$ \\
\hline Normal LD & & $4.2 \pm 0.4$ & $59.9 \pm 12.9$ & 0.007 \\
\hline Normal DD & & $2.3 \pm 0.9$ & $48.5 \pm 4.3$ & $<0.0001$ \\
\hline Reversed LD1 & & $49.4 \pm 2.43$ & $11.8 \pm 1.32$ & $<0.0001$ \\
\hline Reversed DD1 & & $51.0 \pm 2.7$ & $12.0 \pm 1.4$ & $<0.0001$ \\
\hline Reversed LD2 & & $61.2 \pm 4.4$ & $24.3 \pm 2.2$ & $<0.0001$ \\
\hline Reversed DD2 & & $55.7 \pm 6.3$ & $5.6 \pm 3.2$ & $<0.0001$ \\
\hline
\end{tabular}

Summary of experiments testing the influence of the light/dark cycle and feeding time on the level of neuronal proliferation. The light/dark and feeding regimes and resulting mean number $( \pm$ SEM) of BrdU-labeled profiles during the dusk and dawn periods are shown. White bars indicate lights on and black bars indicate lights off. Asterisks mark the feeding time.

placed in total darkness ("reversed DD1") for 3 days maintained this reversed LD pattern of proliferation, with peak neurogenesis at subjective dusk (Table 1). These data suggest that the diurnal rhythm of neurogenesis observed is a product of an endogenous circadian oscillator that is entrained by the light/dark cycle.

To separate the influences of feeding and light on the patterns of neurogenesis observed, a third group of lobsters was entrained to the reversed LD conditions for a period of 2 weeks. However, the feeding time for these animals was maintained at clock hour 16:3019:30 (see Fig. 2), the feeding time utilized for the normal LD group. In this experiment, therefore, the light cycle was shifted by $12 \mathrm{~h}$ relative to the original normal LD animals, but the feeding time was not shifted with the light cycle ("reversed LD2"). As in prior experiments, BrdU labeling showed that the major peak of neurogenesis was in the $3 \mathrm{~h}$ surrounding subjective dusk (Table 1). Some neurogenesis was again observed at subjective dawn; however, the peak was significantly larger than in the previous reversed LD1 experiment for the same time period [24.3 \pm 2.2 (LD2) vs. $11.8 \pm 1.32$ (LD1) BrdU-labeled profiles; $p$ $<$.001]. When animals from the reversed LD2 group were subjected to total darkness for 3 days, the peak of neurogenesis at subjective dusk was maintained (Table 1). However, the peak at subjective dawn was reduced by $77 \%$ in DD2 (Table 1), suggesting that some of the proliferation was induced by feeding.

These data indicate that the diurnal rhythm of neurogenesis is due to an endogenous oscillation that is entrained by light. Feeding appears to serve as a second stimulus for neurogenesis, however, the extent of this influence has not been fully examined. The increase in neuronal proliferation due to feeding may be related to activity induced by food availability, as activity rhythms are often correlated with foraging behavior (Cooper and Uzmann, 1980). Chemosensory stimulation also may play a role in these events by influencing entrainment of circadian rhythms (Amir et al., 1999).

\section{DISCUSSION}

Circadian control of cell division is well established. Diurnal variation in cell proliferation has been documented in rodent and human studies of bone marrow (Smalland et al., 2002), gastrointestinal tract mucosa (Marra et al., 1994), epidermis (Brown, 1991), liver (Haus and Halberg, 1966), and cornea (Scheving and Pauly, 1967). However, our demonstration of circadian control of the neuronal cell cycle is the first example of light entrainment of neurogenesis (Bagay et al., 2001).

The highest level of neurogenesis that we observe occurs at dusk, which is the time of maximal activity for lobsters (Cooper and Uzmann, 1980; Chabot et al., 2001), which are nocturnal. Although light can phaseshift the primary peak of neuronal proliferation, it is clear from our studies that time of feeding also can influence the timing and magnitude of neurogenesis (Table 1). However, light and feeding are not the only factors that influence neurogenesis in the olfactory pathway of crustaceans. Sandeman and Sandeman (2000) have shown that isolation and confinement of juvenile crayfish in a restricted area results in a lower rate of proliferation and survival of local and projec- 
tion neurons in the olfactory pathway when compared with their siblings that were allowed to live together in a relatively unrestricted area. These data suggest that differences in neuronal proliferation and survival are due to variables of space and/or social interaction. Their studies, which were modeled after those of Kemperman and Gage (1999) in mice, illustrate that environmental (activity, social) influences on neurogenesis are not confined to mammalian systems, but are found across phylogenetically diverse species. The fact that increased physical activity has been shown to enhance levels of neurogenesis in mice (Van Praag et al., 1999) is of particular interest in light of the present study, as locomotory activity is generally under circadian control.

Serotonin also is a regulator of neurogenesis in vertebrate (Gould et al., 1999; Brezun and Daszuta, 2000) and invertebrate (Beltz et al., 2001; Benton and Beltz, 2002) species. Studies in the lobster suggest that serotonin levels influence the proliferation and survival of projection neurons in the olfactory pathway (Beltz et al., 2001), the same group of neurons examined in the present study. These results mirror findings in the mammalian brain, where increased serotonin stimulates neuronal proliferation in the adult hippocampus (Brezun and Daszuta, 2000). It is of particular interest in the context of the findings presented in this article, that serotonin levels fluctuate on a circadian cycle in many organisms (CastanonCervantes et al., 1999; Grimes et al., 2000), suggesting serotonergic regulation of rhythmicity downstream from the primary circadian oscillator.

The dentate gyrus of the hippocampus is one of the primary sites of persistent neurogenesis in adult mammals. One current theory of depression proposes that the waning and waxing of neurogenesis in the hippocampus are causally related to the onset of, and recovery from, episodes of this disease (Jacobs et al., 2000). Since new neurons are generated throughout life in both vertebrate and invertebrate organisms, knowledge of disease mechanisms in humans may be enlightened by studies of model systems such as the crustacean brain, where neurogenesis can be tightly controlled with a variety of environmental and hormonal factors. Because mechanisms regulating neurogenesis are conserved across a range of phylogenetically diverse species, our data raise the possibility that light-controlled rhythms are a primary regulator of neurogenesis in a variety of organisms, and that hormonal and activity-driven influences over neuronal proliferation are secondary events in this complex circadian control pathway. It is therefore intriguing to speculate that the timing and/or duration of light exposure may be intimately related to the expression of certain forms of depressive illness, such as seasonal affective disorder.

The authors thank P. Carey and V. Quinan for technical assistance; F. Berthiaume, S. J. Kohler, and DeF. Mellon for critical readings of this manuscript; and the New England Aquarium for providing juvenile lobsters.

\section{REFERENCES}

Aiken DE. 1973. Proecdysis, setal development, and molt prediction in the American lobster (Homarus americanus). J Fish Res Board Can 30:1337-1344.

Altman J, Das GD. 1965. Autoradiographic and histological evidence of postnatal hippocampal neurogenesis in rats. J Comp Neurol 124:319-335.

Alvarez-Buylla A, Kirn JR, Nottebohm F. 1990. Birth of projection neurons in adult avian brain may be related to perceptual or motor learning. Science 249:1444-1446.

Amir S, Cain S, Sullivan J, Robinson B, Stewart J. 1999. In rats, odor-induced Fos in the olfactory pathway depends on the phase of the circadian clock. J Neurosci Lett 272:175-178.

Bagay L, Rehm K, Benton J, Beltz B. 2001. Circadian control of neurogenesis in the lobster brain. Soc Neurosci Abstr 27:587.9.

Beltz BS, Benton JL, Sullivan JM. 2001. Transient uptake of serotonin by newborn olfactory projection neurons. Proc Natl Acad Sci USA 22:12730-12735.

Benton JL, Beltz BS. 2002. Patterns of neurogenesis in the midbrain of embryonic lobsters differs from proliferation in the insect and crustacean ventral nerve cord. J Neurobiol, In press.

Brezun JM, Daszuta A. 2000. Serotonin may stimulate granule cell proliferation in the adult hippocampus, as observed in rats grafted with fetal raphe neurons. Eur J Neurosci 12:391-396.

Brown W. 1991. A review and mathematical analysis of circadian rhythms in cell proliferation in mouse, rat and human epidermis. J Invest Dermatol 97:273-280.

Castanon-Cervantes O, Battelle B, Fanjul-Moles ML. 1999. Rhythmic changes in serotonin content of the brain and eyestalk of crayfish during development. J Exp Biol 202: 2823-2830.

Cayre M, Strambi C, Charpin P, Augier R, Meyer MR, Edwards JS, Strambi A. 1996. Neurogenesis in adult insect mushroom bodies. J Comp Neurol 371:300-310.

Chabot CC, Gallagher JW, Greene BM. 2001. Daily and circadian modulation of locomotion and heart rate in the lobster, Homarus americanus. Soc Neurosci Abstr 29: 185.8 .

Cooper RA, Uzmann JR. 1980. Ecology of juvenile and adult Homarus. In: The biology and management of lobsters. Vol 2. Ecology and management p 97-142. New York: Academic Press.

Eriksson PS, Perfilieva E, Bjork-Eriksson T, Alborn AM, 
Nordborg C, Peterson DA, Gage FH. 1998. Neurogenesis in the adult human hippocampus. Nat Med 4:1313-1317.

Gould E. 1999. Serotonin and hippocampal neurogenesis. Neuropsychopharmacology 21:46S-51S.

Gould E, Reeves AJ, Graziano MSA, Gross CG. 1999. Neurogenesis in the neocortex of adult primates. Science 286:548-552.

Graziadei PPC, Monti Graziadei GA. 1986. Principles of organization of the vertebrate olfactory glomerulus: an hypothesis. Neuroscience 19:1025-1035.

Grimes MA, Cameron JL, Fernstrom JD. 2000. Cerebrospinal fluid concentrations of tryptophan and 5-hydroxyindoleacetic acid in Macaca mulatta: diurnal variations and response to chronic changes in dietary protein intake. Neurochem Res 25:413-422.

Harrison P, Cate H, Swanson E, Derby C. 2001. Postembryonic proliferation in the spiny lobster antennular epithelium: rate of genesis of olfactory receptor neurons is dependent on molt stage. J Neurobiol 47:51-66.

Harzsch S, Dawirs RR. 1996. Neurogenesis in the developing crab brain: postembryonic generation of neurons persists beyond metamorphosis. J Neurobiol 29:384-398.

Harzsch S, Miller J, Benton JL, Beltz BS. 1999. From embryo to adult: persistent neurogenesis and apoptotic cell death shape the lobster deutocerebrum. J Neurosci 19:3472-3485.

Haus E, Halberg F. 1966. Persisting circadian rhythm in hepatic glycogen of mice during inanition and dehydration. Experientia 22:113-114.

Jacobs BL, Praag H, Gage FH. 2000. Adult brain neurogenesis and psychiatry: a novel theory of depression. Mol Psychiatry 5:262-269.

Kemperman G, Gage FH. 1999. New nerve cells for the adult brain. Sci Am 280:48-53.

Kornack DR, Rakic P. 2001. Cell proliferation without neurogenesis in adult primate neocortex. Science 294: 2127-2130.

Lois C, Alvarez-Buylla A. 1994. Long distance neuronal migration in the adult mammalian brain. Science 264 : $1145-1148$.

Marra G, Anti M, Percesepe A, Armelao F, Ficarelli R,
Coco C, Rinelli A, Vecchio F, D’Arcangelo E. 1994. Circadian variations of epithelial cell proliferation in human rectal crypts. Gastroenterology 106:982-987.

Rasika S, Nottebohm F, Alvarez-Buylla A. 1994. Testosterone increases the recruitment and/or survival of new high vocal center neurons in adult female canaries. Proc Natl Acad Sci USA 91:7854-7858.

Sandeman DC, Sandeman RE. 1994. Electrical responses and synaptic connections of giant serotonin-immunoreactive neurons in crayfish olfactory and accessory lobes. J Comp Neurol 341:130-144.

Sandeman D, Beltz BS, Sandeman R. 1995. Crayfish brain interneurons that converge with serotonin giant cells in accessory lobe glomeruli. J Comp Neurol 352:263-279.

Sandeman R, Sandeman D. 2000. "Impoverished" and "enriched" living conditions influence the proliferation and survival of neurons in crayfish brain. J Neurobiol 45:215226.

Sandeman R, Clarke D, Sandeman D, Manly M. 1998. Growth-related and antennular amputation-induced changes in the olfactory centers of crayfish brain. J Neurosci 18:6195-6206.

Smaaland R, Sothern RB, Laerum OD, Abrahamsen JF. 2002. Rhythms in human bone marrow and blood cells. Chronobiol Int 1:101-127.

Scheving L, Pauly J. 1967. Circadian phase relationships of thymidine- ${ }^{3} \mathrm{H}$ uptake, labeled nuclei, grain counts, and cell division rate in rat corneal epithelium. J Cell Biol 32:677-683.

Schmidt M. 2001. Neuronal differentiation and long-term survival of newly generated cells in the olfactory midbrain of the adult spiny lobster, Panulirus argus. J Neurobiol 48:181-203.

Strausfeld NJ, Hildebrand JG. 1999. Olfactory systems: common design, uncommon origins? Curr Opin Neurobiol 5:634-639.

Van Praag H, Christie BR, Sejnowski TJ, Gage FH. 1999. Running enhances neurogenesis, learning and long-term potentiation in mice. Proc Natl Acad Sci USA 96:1342713431. 